\title{
An estimate of the inflation factor and analysis sensitivity in the ensemble Kalman filter
}

\author{
Guocan $\mathrm{Wu}^{1,2}$ and Xiaogu Zheng ${ }^{3}$ \\ ${ }^{1}$ College of Global Change and Earth System Science, Beijing Normal University, Beijing, China \\ ${ }^{2}$ Joint Center for Global Change Studies, Beijing, China \\ ${ }^{3}$ Key Laboratory of Regional Climate-Environment Research for East Asia, Institute of Atmospheric Physics, \\ Chinese Academy of Sciences, Beijing, China
}

Correspondence to: Guocan Wu (gcwu@bnu.edu.cn)

Received: 18 August 2016 - Discussion started: 4 October 2016

Revised: 17 May 2017 - Accepted: 26 May 2017 - Published: 3 July 2017

\begin{abstract}
The ensemble Kalman filter (EnKF) is a widely used ensemble-based assimilation method, which estimates the forecast error covariance matrix using a Monte Carlo approach that involves an ensemble of short-term forecasts. While the accuracy of the forecast error covariance matrix is crucial for achieving accurate forecasts, the estimate given by the EnKF needs to be improved using inflation techniques. Otherwise, the sampling covariance matrix of perturbed forecast states will underestimate the true forecast error covariance matrix because of the limited ensemble size and large model errors, which may eventually result in the divergence of the filter.

In this study, the forecast error covariance inflation factor is estimated using a generalized cross-validation technique. The improved EnKF assimilation scheme is tested on the atmosphere-like Lorenz-96 model with spatially correlated observations, and is shown to reduce the analysis error and increase its sensitivity to the observations.
\end{abstract}

\section{Introduction}

For state variables in geophysical research fields, a common assumption is that systems have "true" underlying states. Data assimilation is a powerful mechanism for estimating the true trajectory based on the effective combination of a dynamic forecast system (such as a numerical model) and observations (Miller et al., 1994). Data assimilation provides an analysis state that is usually a better estimate of the state variable because it considers all of the information provided by the model forecasts and observations. In fact, the analysis state can generally be treated as the weighted average of the model forecasts and observations, while the weights are approximately proportional to the inverse of the corresponding covariance matrices (Talagrand, 1997). Therefore, the performance of a data assimilation method relies significantly on whether the error covariance matrices are estimated accurately. If this is the case, the assimilation can be accomplished with the rapid development of supercomputers (Reichle, 2008), although finding the appropriate analysis state is a much difficult problem when the models are nonlinear.

The ensemble Kalman filter (EnKF) is a practical ensemble-based assimilation scheme that estimates the forecast error covariance matrix using a Monte Carlo method with the short-term ensemble forecast states (Burgers et al., 1998; Evensen, 1994). Because of the limited ensemble size and large model errors, the sampling covariance matrix of the ensemble forecast states usually underestimates the true forecast error covariance matrix. This finding indicates that the filter is over reliant on the model forecasts and excludes the observations. It can eventually result in the divergence of the filter (Anderson and Anderson, 1999; Constantinescu et al., 2007; Wu et al., 2014).

The covariance inflation technique is used to mitigate filter divergence by inflating the empirical covariance in EnKF, and it can increase the weight of the observations in the analysis state (Xu et al., 2013). In reality, this method will perturb the subspace spanned by the ensemble vectors and better capture the sub-growing directions that may not have been captured by the original ensemble (Yang et al., 2015). Therefore, 
using the inflation technique to enhance the estimate accuracy of the forecast error covariance matrix is increasingly important.

A widely used inflation technique involves multiplying the forecast error matrix by an inflation factor, which must be chosen appropriately. In early studies, researchers usually tuned the inflation factor by repeated assimilation experiments and selected the estimated inflation factor according to their experience and prior knowledge (Anderson and Anderson, 1999). However, such methods are very empirical and subjective. It is not appropriate to use the same inflation factor during all the assimilation procedure. Too small or too large an inflation factor will cause the analysis state to over rely on the model forecasts or observations, and can seriously undermine the accuracy and stability of the filter.

In later studies, the inflation factor is estimated online based on the innovation statistic (observation-minusforecast; Dee, 1995; Dee and Silva, 1999) with different conditions. Moment estimation can facilitate the calculation by solving an equation of the innovation statistic and its realization (Li et al., 2009; Miyoshi, 2011; Wang and Bishop, 2003). Maximum likelihood approach can obtain a better estimate of the inflation factor than moment approach, although it must calculate a high-dimensional matrix determinant (Liang et al., 2012; Zheng, 2009). Bayesian approach assumes a prior distribution for the inflation factor but is limited by spatially independent observational errors (Anderson, 2007, 2009). This study seeks to address the estimation of the inflation factor from the perspective of cross-validation (CV).

The concept of CV was first introduced for linear regressions (Allen, 1974) and spline smoothing (Wahba and Wold, 1975), and it represents a common approach that can be applied to estimate tuning parameters in generalized additive models, nonparametric regressions and kernel smoothing (Eubank, 1999; Gentle et al., 2004; Green and Silverman, 1994; Wand and Jones, 1995). Usually, the data are divided into subsets some of which are used for modeling and analysis while others for verification and validation. The most widely used technique removes only one data point and uses the remainder to estimate the value at this point to test the estimation accuracy, which is also called the leave-one-out cross-validation (Gu and Wahba, 1991).

The basic motivation behind CV is to minimize the prediction error at the sampling points. The generalized crossvalidation $(\mathrm{GCV})$ is a modified form of ordinary $\mathrm{CV}$, that has been found to possess several favorable properties and is more popular for selecting tuning parameters (Craven and Wahba, 1979). For instance, Gu and Wahba (1991) applied the Newton's method to optimize the GCV score with multiple smoothing parameters in a smoothing spline model. Wahba et al. (1995) briefly reviewed the properties of the GCV and conducted an experiment to choose smoothing parameters in the context of variational data assimilation schemes with numerical weather prediction models. Zheng and Basher (1995) also applied the GCV in a thin-plate smoothing spline model of spatial climate data to deal with South Pacific rainfalls.

Actually, the GCV criterion is based on a predictive meansquare-error criterion that attempts to obtain a best estimate (Wahba et al., 1995). It has a rotation-invariant property that is relative to the orthogonal transformation of the observations and is a consistent estimate of the relative loss $(\mathrm{Gu}$, 2002). For the inverse problems in such fields as meteorological data assimilation, GCV method can choose parameters systematically by minimizing a given objective function that will improve the assimilation results. It can particularly select parameters that reflect not only measurement accuracies from different sources but also model capability (Krakauer et al., 2004).

This study proposes a new method for choosing the inflation factor using GCV method. The suitability of this choice is assessed using a statistic known as the analysis sensitivity, which apportions uncertainty in the output to different sources of uncertainty in the input (Saltelli et al., 2004, 2008). In the context of statistical data assimilation, this quantity describes the sensitivity of the analysis to the observations, which is complementary to the sensitivity of the analysis to model forecasts (Cardinali et al., 2004; Liu et al., 2009).

This study focuses on a methodology that can be potentially applied to geophysical applications of data assimilation in the near future. This paper consists of four sections. The conventional EnKF scheme is summarized and the improved EnKF with GCV inflation scheme is proposed in Sect. 2, the verification and validation processes are conducted on an idealized model in Sect. 3, the discussions are presented in Sect. 4 and conclusions are given in Sect. 5.

\section{Methodology}

\subsection{EnKF algorithm}

For consistency, a nonlinear discrete-time dynamical forecast model and linear observation system can be expressed as follows (Ide et al., 1997):

$\boldsymbol{x}_{i}^{\mathrm{t}}=M_{i-1}\left(\boldsymbol{x}_{i-1}^{\mathrm{a}}\right)+\eta_{i}$,

$\boldsymbol{y}_{i}^{\mathrm{o}}=\mathbf{H}_{i} \boldsymbol{x}_{i}^{\mathrm{t}}+\boldsymbol{\varepsilon}_{i}$,

where $i$ represents the time index; $\boldsymbol{x}_{i}^{\mathrm{t}}=\left\{x_{i, 1}^{\mathrm{t}}, x_{i, 2}^{\mathrm{t}}, \ldots, x_{i, n}^{\mathrm{t}}\right\}^{\mathrm{T}}$ represents the $n$-dimensional true state vector at the $i$ th time step; $\boldsymbol{x}_{i-1}^{\mathrm{a}}=\left\{x_{i-1,1}^{\mathrm{a}}, x_{i-1,2}^{\mathrm{a}}, \ldots, x_{i-1, n}^{\mathrm{a}}\right\}^{\mathrm{T}}$ represents the $n$ dimensional analysis state vector, which is an estimate of $\boldsymbol{x}_{i-1}^{\mathrm{t}} ; M_{i-1}$ represents a nonlinear dynamical forecast operator such as a numerical weather prediction model; $\boldsymbol{y}_{i}^{\mathrm{o}}=$ $\left\{y_{i, 1}^{\mathrm{o}}, y_{i, 2}^{\mathrm{o}}, \ldots, y_{i, p_{i}}^{\mathrm{o}}\right\}^{\mathrm{T}}$ represents a $p_{i}$-dimensional observation vector; $\mathbf{H}_{i}$ represents the observation operator matrix; and $\boldsymbol{\eta}_{i}$ and $\boldsymbol{\varepsilon}_{i}$ represent the forecast and observation error 
vectors, which are assumed to be time uncorrelated, statistically independent of each other and have mean zero and covariance matrices $\mathbf{P}_{i}$ and $\mathbf{R}_{i}$, respectively. The EnKF assimilation result is a series of analysis states $\boldsymbol{x}_{i}^{\mathrm{a}}$ that is an accurate estimate of the corresponding true states $\boldsymbol{x}_{i}^{\mathrm{t}}$ based on the information provided by $M_{i}$ and $\boldsymbol{y}_{i}^{\mathrm{o}}$.

Suppose the perturbed analysis state at a previous time step $\boldsymbol{x}_{i-1}^{\mathrm{a}(j)}$ has been estimated $(1 \leq j \leq m$ and $m$ is the ensemble size), the detailed EnKF assimilation procedure is summarized as the following forecast step and analysis step (Burgers et al., 1998; Evensen, 1994).

\subsubsection{Step 1: forecast step}

The perturbed forecast states are generated by running dynamical model forward:

$\boldsymbol{x}_{i}^{\mathrm{f}(j)}=M_{i-1}\left(\boldsymbol{x}_{i-1}^{\mathrm{a}(j)}\right)$.

The forecast state $\boldsymbol{x}_{i}^{\mathrm{f}}$ is defined as the ensemble mean of $\boldsymbol{x}_{i}^{\mathrm{f}(j)}$, and the forecast error covariance matrix is initially estimated as the sampling covariance matrix of perturbed forecast states:

$\mathbf{P}_{i}=\frac{1}{m-1} \sum_{j=1}^{m}\left(\boldsymbol{x}_{i}^{\mathrm{f}(j)}-\boldsymbol{x}_{i}^{\mathrm{f}}\right)\left(\boldsymbol{x}_{i}^{\mathrm{f}(j)}-\boldsymbol{x}_{i}^{\mathrm{f}}\right)^{\mathrm{T}}$.

\subsubsection{Step 2: analysis step}

The analysis state is estimated by minimizing the following cost function:

$$
\begin{aligned}
J(\boldsymbol{x}) & =\left(\boldsymbol{x}-\boldsymbol{x}_{i}^{\mathrm{f}}\right)^{\mathrm{T}} \mathbf{P}_{i}^{-1}\left(\boldsymbol{x}-\boldsymbol{x}_{i}^{\mathrm{f}}\right) \\
& +\left(\boldsymbol{y}_{i}^{\mathrm{o}}-\mathbf{H}_{i} \boldsymbol{x}\right)^{\mathrm{T}} \mathbf{R}_{i}^{-1}\left(\boldsymbol{y}_{i}^{\mathrm{o}}-\mathbf{H}_{i} \boldsymbol{x}\right),
\end{aligned}
$$

which has the analytic form

$\boldsymbol{x}_{i}^{\mathrm{a}}=\boldsymbol{x}_{i}^{\mathrm{f}}+\mathbf{P}_{i} \mathbf{H}_{i}^{\mathrm{T}}\left(\mathbf{H}_{i} \mathbf{P}_{i} \mathbf{H}_{i}^{\mathrm{T}}+\mathbf{R}_{i}\right)^{-1} \boldsymbol{d}_{i}$,

where

$\boldsymbol{d}_{i}=\boldsymbol{y}_{i}^{\mathrm{o}}-\mathbf{H}_{i} \boldsymbol{x}_{i}^{\mathrm{f}}$

is the innovation statistic (observation-minus-forecast residual in observation space). To complete the ensemble forecast, the perturbed analysis states are calculated using perturbed observations (Burgers et al., 1998):

$\boldsymbol{x}_{i}^{\mathrm{a}(j)}=\boldsymbol{x}_{i}^{\mathrm{f}(j)}+\mathbf{P}_{i} \mathbf{H}_{i}^{\mathrm{T}}\left(\mathbf{H}_{i} \mathbf{P}_{i} \mathbf{H}_{i}^{\mathrm{T}}+\mathbf{R}_{i}\right)^{-1}\left(\boldsymbol{d}_{i}+\boldsymbol{\varepsilon}_{i}^{\prime(\mathrm{j})}\right)$,

where $\boldsymbol{\varepsilon}_{i}^{\prime(\mathrm{j})}$ is a normally distributed random variable with mean zero and covariance matrix $\mathbf{R}_{i}$. Here, $\left(\mathbf{H}_{i} \mathbf{P}_{i} \mathbf{H}_{i}^{\mathrm{T}}+\mathbf{R}_{i}\right)^{-1}$ can be easily calculated using the Sherman-Morrison-Woodbury formula (Golub and Loan, 1996; Liang et al., 2012; Tippett et al., 2003). Finally, set $i=i+1$, return to Step 1 for the model forecast at the next time step and repeat until the model reaches the last time step $N$.

\subsection{Influence matrix and forecast error inflation}

The forecast error inflation procedure should be added to any ensemble-based assimilation scheme to prevent the filter from diverging (Anderson and Anderson, 1999; Constantinescu et al., 2007). Multiplicative inflation is one of the commonly used inflation techniques, and it adjusts the initially estimated forecast error covariance matrix $\mathbf{P}_{i}$ to $\lambda_{i} \mathbf{P}_{i}$ after estimating the inflation factors $\lambda_{i}$ properly.

In this study, a new procedure for estimating multiplicative inflation factors $\lambda_{i}$ is proposed based on the following GCV function (Craven and Wahba, 1979)

$\operatorname{GCV}_{i}(\lambda)=\frac{\frac{1}{p_{i}} \boldsymbol{d}_{i}^{\mathrm{T}} \mathbf{R}_{i}^{-1 / 2}\left(\mathbf{I}_{p_{i}}-\mathbf{A}_{i}(\lambda)\right)^{2} \mathbf{R}_{i}^{-1 / 2} \boldsymbol{d}_{i}}{\left[\frac{1}{p_{i}} \operatorname{Tr}\left(\mathbf{I}_{p_{i}}-\mathbf{A}_{i}(\lambda)\right)\right]^{2}}$,

where $\mathbf{I}_{p_{i}}$ is the identity matrix with dimension $p_{i} \times p_{i}$; $\mathbf{R}_{i}^{-1 / 2}$ is the square root matrix of $\mathbf{R}_{i}$; and

$\mathbf{A}_{i}(\lambda)=\mathbf{I}_{p_{i}}-\mathbf{R}_{i}^{1 / 2}\left(\mathbf{H}_{i} \lambda \mathbf{P}_{i} \mathbf{H}_{i}^{\mathrm{T}}+\mathbf{R}_{i}\right)^{-1} \mathbf{R}_{i}^{1 / 2}$

is the influence matrix (see Appendix A for details).

The inflation factor $\lambda_{i}$ is estimated by minimizing the GCV (Eq. 9) as an objective function, and it is implemented between steps 1 and 2 in Sect. 2.1. Then, the perturbed analysis states are modified to

$\boldsymbol{x}_{i}^{\mathrm{a}(\mathrm{j})}=\boldsymbol{x}_{i}^{\mathrm{f}(\mathrm{j})}+\lambda_{i} \mathbf{P}_{i} \mathbf{H}_{i}^{\mathrm{T}}\left(\mathbf{H}_{i} \lambda_{i} \mathbf{P}_{i} \mathbf{H}_{i}^{\mathrm{T}}+\mathbf{R}_{i}\right)^{-1}\left(\boldsymbol{d}_{i}+\boldsymbol{\varepsilon}_{i}^{\prime(j)}\right)$.

The flowchart of the EnKF equipped with the proposed forecast error inflation based on the GCV method is shown in Fig. 1.

\subsection{Analysis sensitivity}

In the EnKF, the analysis state (Eq. 6) is a weighted average of the observation and forecast. That is

$\boldsymbol{x}_{i}^{\mathrm{a}}=\mathbf{K}_{i} \boldsymbol{y}_{i}^{\mathrm{o}}+\left(\mathbf{I}_{n}-\mathbf{K}_{i} \mathbf{H}_{i}\right) \boldsymbol{x}_{i}^{\mathrm{f}}$,

where $\mathbf{K}_{i}=\mathbf{P}_{i} \mathbf{H}_{i}^{\mathrm{T}}\left(\mathbf{H}_{i} \mathbf{P}_{i} \mathbf{H}_{i}^{\mathrm{T}}+\mathbf{R}_{i}\right)^{-1}$ is the Kalman gain matrix and $\mathbf{I}_{n}$ is the identity matrix with dimension $n \times n$. Then, the normalized analysis vector can be expressed as follows:

$\widetilde{\boldsymbol{y}}_{i}^{\mathrm{a}}=\mathbf{R}_{i}^{-1 / 2} \mathbf{H}_{i} \mathbf{K}_{i} \mathbf{R}_{i}^{1 / 2} \widetilde{\boldsymbol{y}}_{i}^{\mathrm{o}}+\mathbf{R}_{i}^{-1 / 2}\left(\mathbf{I}_{p_{i}}-\mathbf{H}_{i} \mathbf{K}_{i}\right) \mathbf{R}_{i}^{1 / 2} \widetilde{\boldsymbol{y}}_{i}^{\mathrm{f}}$,

where $\tilde{\boldsymbol{y}}_{i}^{\mathrm{f}}=\mathbf{R}_{i}^{-1 / 2} \mathbf{H}_{i} \boldsymbol{x}_{i}^{\mathrm{f}}$ is the normalized projection of the forecast on the observation space. The sensitivities of the analysis to the observation and forecast are defined by Eqs. (14) and (15), respectively:

$\mathbf{S}_{i}^{\mathrm{o}}=\frac{\partial \widetilde{\boldsymbol{y}}_{i}^{\mathrm{a}}}{\partial \widetilde{\boldsymbol{y}}_{i}^{\mathrm{o}}}=\mathbf{R}_{i}^{1 / 2} \mathbf{K}_{i}^{\mathrm{T}} \mathbf{H}_{i}^{\mathrm{T}} \mathbf{R}_{i}^{-1 / 2}$,

$\mathbf{S}_{i}^{\mathrm{f}}=\frac{\partial \widetilde{\boldsymbol{y}}_{i}^{\mathrm{a}}}{\partial \widetilde{\boldsymbol{y}}_{i}^{\mathrm{f}}}=\mathbf{R}_{i}^{1 / 2}\left(\mathbf{I}_{p_{i}}-\mathbf{K}_{i}^{\mathrm{T}} \mathbf{H}_{i}^{\mathrm{T}}\right) \mathbf{R}_{i}^{-1 / 2}$,

which satisfy $\mathbf{S}_{i}^{\mathrm{o}}+\mathbf{S}_{i}^{\mathrm{f}}=\mathbf{I}_{p_{i}}$. 


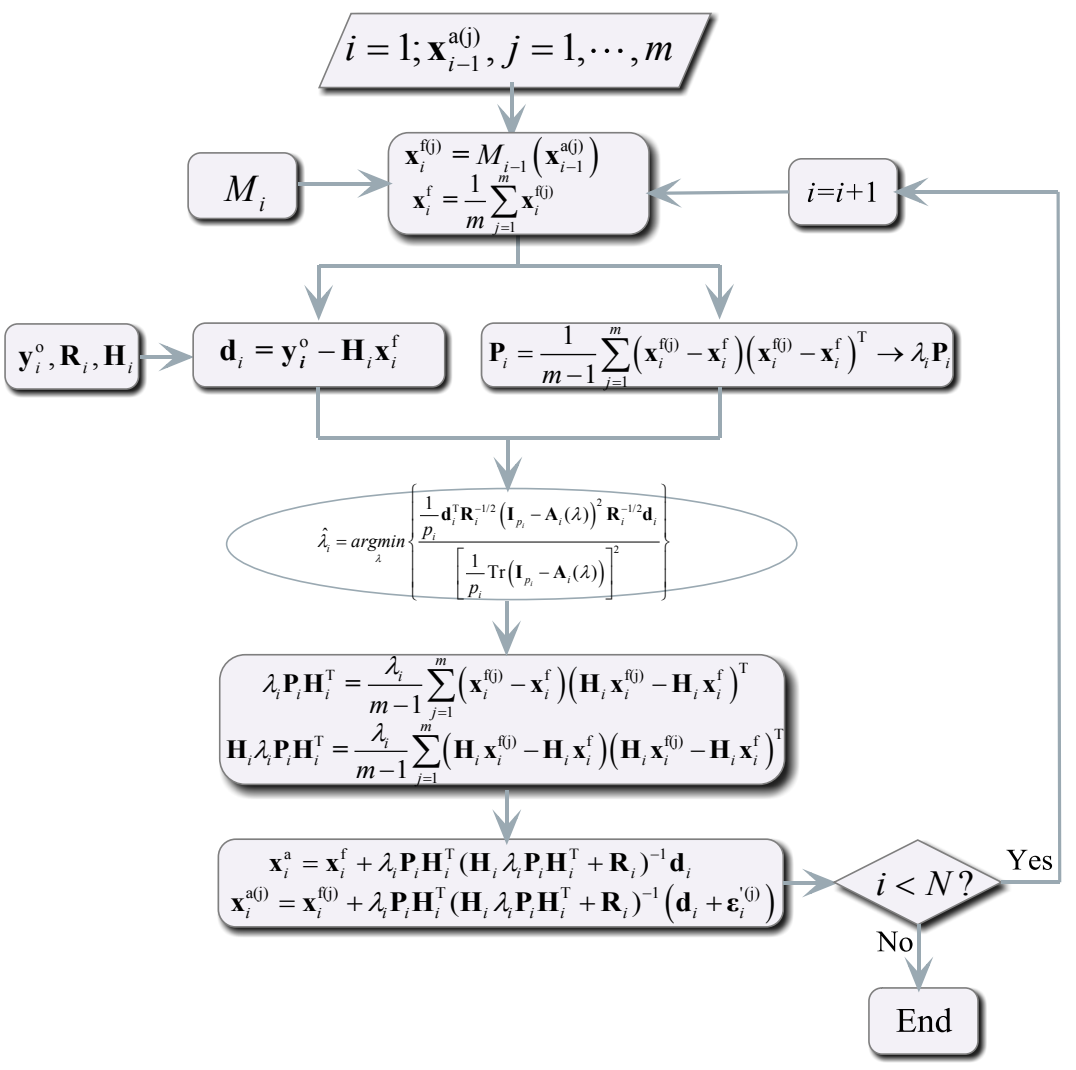

Figure 1. Flowchart of the proposed assimilation scheme.

The elements of the matrix $\mathbf{S}_{i}^{\mathrm{o}}$ reflect the sensitivity of the normalized analysis state to the normalized observations; its diagonal elements are the analysis self-sensitivities and the off-diagonal elements are the cross-sensitivities. On the other hand, the elements of the matrix $\mathbf{S}_{i}^{\mathrm{f}}$ reflect the sensitivity of the normalized analysis state to the normalized forecast state. The two quantities are complementary, and the GCV function can be interpreted as minimizing the normalized forecast sensitivity because the inflation scheme will increase the observation weight appropriately.

In fact, the sensitivity matrix $\mathbf{S}_{i}^{\mathbf{o}}$ is equal to the influence matrix $\mathbf{A}_{i}$ (see Appendix B for detailed proof), whose trace can be used to measure the "equivalent number of parameters" or "degrees of freedom for the signal" (Gu, 2002; Pena and Yohai, 1991). Similarly, the sensitivity matrix $\mathbf{S}_{i}^{\text {o can be }}$ interpreted as a measurement of the amount of information extracted from the observations (Ellison et al., 2009). Trace diagnostics can be used to analyze the sensitivities to observations or forecast vectors (Cardinali et al., 2004). The global average influence (GAI) at the $i$ th time step is defined as the globally averaged observation influence:

$\mathrm{GAI}=\frac{\operatorname{Tr}\left(\mathbf{S}_{i}^{\mathrm{o}}\right)}{p_{i}}$, where $p_{i}$ is the total number of observations at the $i$ th time step.

In the conventional EnKF, the forecast error covariance matrix $\mathbf{P}_{i}$ is initially estimated using a Monte Carlo method with short-term ensemble forecast states. However, because of the limited ensemble size and large model errors, the sampling covariance matrix of perturbed forecast states usually underestimate the true forecast error covariance matrix. This will cause the analysis to over rely on the forecast state and exclude useful information from the observations. This is captured by the fact that the GAI values are rather small for the conventional EnKF scheme. Adjusting the inflation of the forecast error covariance matrix alleviates this problem to some extent, as will be shown in the following simulations.

\subsection{Forecast ensemble spread and analysis RMSE}

The spread of the forecast ensemble at the $i$ th step is defined as follows:

Spread $=\sqrt{\frac{1}{n(m-1)} \sum_{j=1}^{m}\left\|\boldsymbol{x}_{i}^{\mathrm{f}(j)}-\boldsymbol{x}_{i}^{\mathrm{f}}\right\|^{2}}$.

Roughly speaking, the forecast ensemble spread is usually underestimated for the conventional EnKF, which also dramatically decreases until the observations ultimately have an 
irrelevant impact on the analysis states. The inflation technique can effectively compensate for the underestimation of the forecast ensemble spread, and thereby can improve the assimilation results.

In the following experiments, the "true" state $\boldsymbol{x}_{i}^{\mathrm{t}}$ is nondimensional and can be obtained by a numerical solution of partial differential equations. In this case, the distance of the analysis state to the true state can be defined as the analysis root mean square error (RMSE), which is used to evaluate the accuracy of the assimilation results. The RMSE at the $i$ th time step is defined as follows:

$\mathrm{RMSE}=\sqrt{\frac{1}{n} \sum_{k=1}^{n}\left(\mathrm{x}_{i, k}^{\mathrm{a}}-\mathrm{x}_{i, k}^{\mathrm{t}}\right)^{2} .}$

where $\mathrm{x}_{i, k}^{\mathrm{a}}$ and $\mathrm{x}_{i, k}^{\mathrm{t}}$ are the $k$ th components of the analysis state and true state at the $i$ th time step. In principle, a smaller RMSE indicates a better performance of the assimilation scheme.

\section{Numerical experiments}

The proposed data assimilation scheme was tested using the Lorenz-96 model (Lorenz, 1996) with model errors and a linear observation system as a test bed. The performances of the assimilation schemes described in Sect. 2 were evaluated via the following experiments.

\subsection{Dynamical forecast model and observation systems}

The Lorenz-96 model (Lorenz, 1996) is a quadratic nonlinear dynamical system that has properties relevant to realistic forecast problems and is governed by the equation

$$
\frac{\mathrm{d} \mathbf{X}_{k}}{\mathrm{~d} t}=\left(\mathbf{X}_{k+1}-\mathbf{X}_{k-2}\right) \mathbf{X}_{k-1}-\mathbf{X}_{k}+F,
$$

where $k=1,2, \ldots, 40$. The cyclic boundary conditions $\mathbf{X}_{-1}=\mathbf{X}_{K-1}, \mathbf{X}_{0}=\mathbf{X}_{K}$ and $\mathbf{X}_{K+1}=\mathbf{X}_{1}$ were applied to ensure that Eq. (19) is well defined for all values of $k$. The Lorenz-96 model is "atmosphere-like" because the three terms on the right-hand side of Eq. (19) are analogous to a nonlinear advection-like term, a damping term, and an external forcing term, respectively. The model can be considered representative of an atmospheric quantity (e.g., zonal wind speed) distributed on a latitude circle. Therefore, the Lorenz96 model has been widely used as a test bed to evaluate the performance of assimilation schemes in many studies (Wu et al., 2013).

The true state is derived by a fourth-order Runge-Kutta time integration scheme (Butcher, 2003). The time step for generating the numerical solution was set at 0.05 nondimensional units, which is roughly equivalent to $6 \mathrm{~h}$ in real time, assuming that the characteristic timescale of the dissipation in the atmosphere is 5 days (Lorenz, 1996). The forcing term was set as $F=8$ so that the leading Lyapunov exponent implies an error-doubling time of approximately 8 time steps and the fractal dimension of the attractor was 27.1 (Lorenz and Emanuel, 1998). The initial value was chosen to be $\mathbf{X}_{k}=F$ when $k \neq 20$ and $\mathbf{X}_{20}=1.001 F$.

In this study, the synthetic observations were assumed to be generated by adding random noises that were multivariate normally distributed with mean zero and covariance matrix $\mathbf{R}_{i}$ to the true states. The frequency was every 4 time steps, which can be used to mimic daily observations in practical problems, such as satellite data. The observation errors were assumed to be spatially correlated, which is common in applications involving remote sensing and radiance data. The variance of the observation at each grid point was set to $\sigma_{\mathrm{o}}^{2}=1$, and the covariance of the observations between the $j$ th and $k$ th grid points was as follows:

$\mathbf{R}_{i}(j, k)=\sigma_{\mathrm{o}}^{2} \times 0.5^{\min \{|j-k|, 40-|j-k|\}}$.

\subsection{Assimilation scheme comparison}

Because model errors are inevitable in practical dynamical forecast models, it is reasonable to add model errors to the Lorenz-96 model in the assimilation process. The Lorenz-96 model is a forced dissipative model with a parameter $F$ that controls the strength of the forcing. Modifying the forcing strength $F$ changes the model forecast states considerably. For values of $F$ that are larger than 3, the system is chaotic (Lorenz and Emanuel, 1998). To simulate model errors, the forcing term for the forecast was set to 7, while using $F=8$ to generate the "true" state. The initially selected ensemble size was 30 .

The Lorenz-96 model was run for 2000 time steps, which is equivalent to approximately 500 days in realistic problems. The synthetic observations were assimilated at every grid point and every 4 time steps using the conventional EnKF, the constant inflated EnKF and the improved EnKF schemes for comparisons. The time series of estimated inflation factors are shown in Fig. 2. It can be seen that the estimated inflation factors vary between 1 and 6 in most instances, although the values smaller than 1 are estimated in several assimilation time steps. The median of the estimated inflation factors was 1.88 , which was used as the inflation factor in the constant inflated EnKF scheme. Since the median is a robust and highly efficient statistic of the central tendency, this can ensure a relative fair comparison between the constant inflated EnKF and the improved EnKF schemes.

The forecast ensemble spread of the conventional EnKF, constant inflated EnKF and improved EnKF are plotted in Fig. 3. For the conventional EnKF, because the forecast states usually shrink together, the forecast ensemble spread was quite small and had a mean value of 0.36 . The mean spread value of the improved EnKF was 3.32, which was larger than that of the constant inflated EnKF (3.25). These findings illustrate that the underestimation of forecast ensemble spread can be effectively compensated for by the two EnKF schemes 


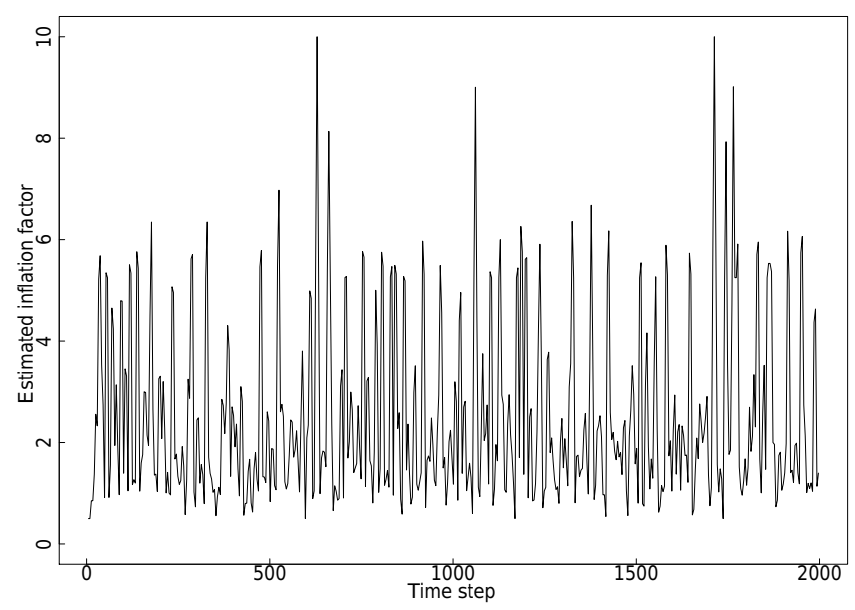

Figure 2. Time series of the estimated inflation factors by minimizing the GCV function. The median of the estimated inflation factors is 1.88 .

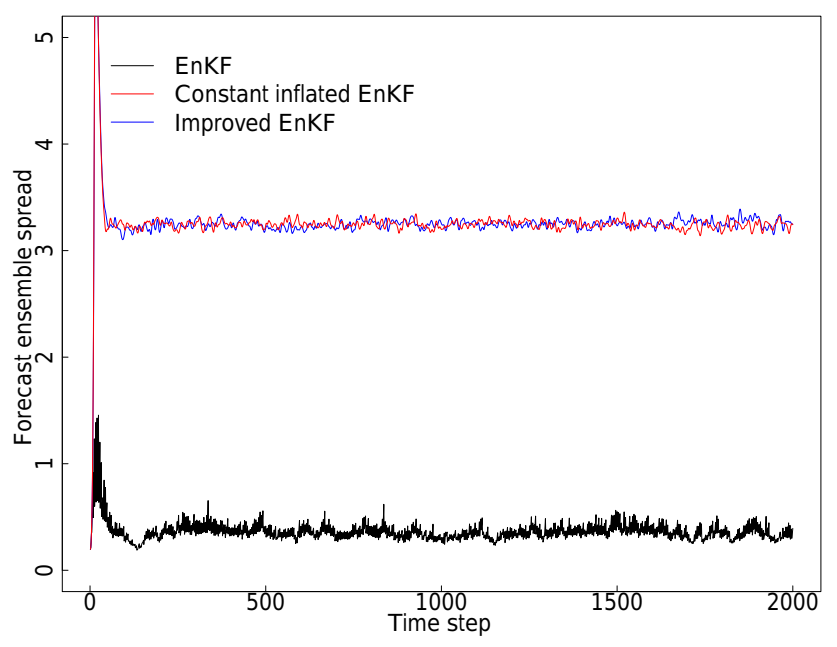

Figure 3. Forecast ensemble spread of the conventional EnKF (black line), the constant inflated EnKF (red line) and the improved EnKF (blue line) for the Lorenz-96 experiment with 40-observation and 30-ensemble member. The constant multiplicative inflation factor is set as 1.88 .

with forecast error inflation and that the improved EnKF is more effective than the constant inflated EnKF.

To evaluate the analysis sensitivity, the GAI statistics (Eq. 16) were calculated, and the results are plotted in Fig. 4. The GAI value increases from $10 \%$ for the conventional EnKF to $30 \%$ for the improved EnKF, indicating that the latter relies more on the observations. This finding is important because the observations can play a significant role in combining the results with the model forecasts to generate the analysis state. In addition to small fluctuations, the mean GAI value of the constant inflated EnKF was $27.80 \%$, which was smaller than that of the improved EnKF.

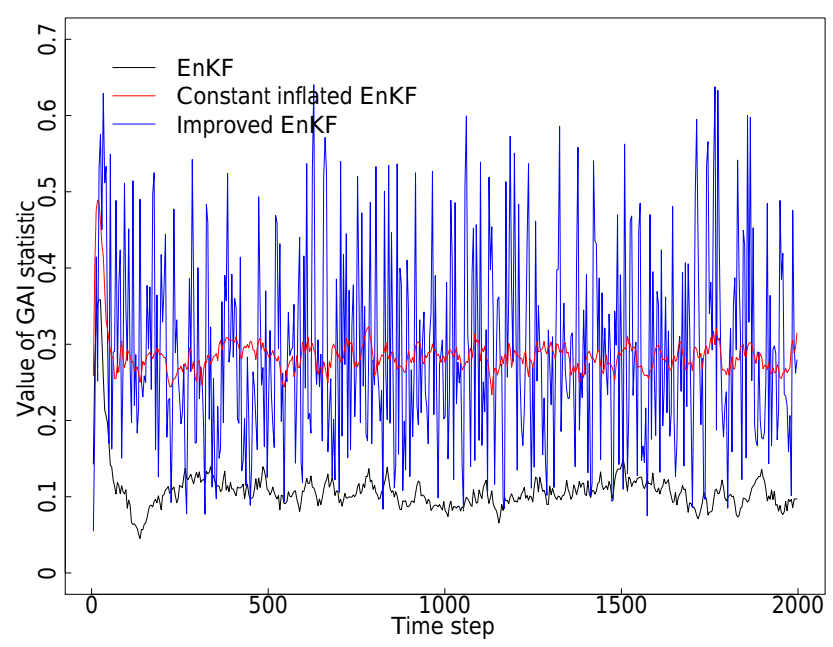

Figure 4. GAI statistics of the conventional EnKF (black line), the constant inflated EnKF (red line) and the improved EnKF (blue line) for the Lorenz-96 experiment with 40-observation and 30-ensemble member. The constant multiplicative inflation factor is set as 1.88 .

To evaluate the analysis estimate accuracy, the analysis RMSE (Eq. 18) and the corresponding values of the GCV functions (Eq. 9) were calculated and plotted in Figs. 5 and 6 , respectively. The results illustrate that the analysis RMSE and the values of the GCV functions decrease sharply for the two EnKF with forecast error inflation schemes. However, the GCV function and the RMSE values of the improved EnKF were about $15 \%$ smaller than those of the constant inflated EnKF, indicating that the online estimate method performs better than the simple multiplicative inflation techniques with a constant value. The correlation coefficient of the analysis RMSE and the value of the GCV function at the assimilation time step were approximately 0.76 , which indicates that the GCV function is a good criterion to estimate the inflation factor.

The ensemble analysis state members of the conventional EnKF, constant inflated EnKF and improved EnKF are shown in Fig. 7, and the results indicate the uncertainty of the analysis state to some extent. The true trajectory obtained by the numerical solution is also plotted. It illustrates that a larger difference occurred between the true trajectory and the ensemble analysis state members for the conventional EnKF than for the improved EnKF and constant inflated EnKF. In addition, the analysis state was more consistent with the true trajectory for the improved EnKF than that for the constant inflated EnKF. Therefore, the GCV inflation can lead to a more accurate analysis state than the simple constant inflation.

The time-mean values of the forecast ensemble spread, the GAI statistics, the GCV functions and the analysis RMSE over 2000 time steps are listed in Table 1. These results illustrate that the forecast error inflation technique using the GCV function performs better than the constant inflated EnKF, 


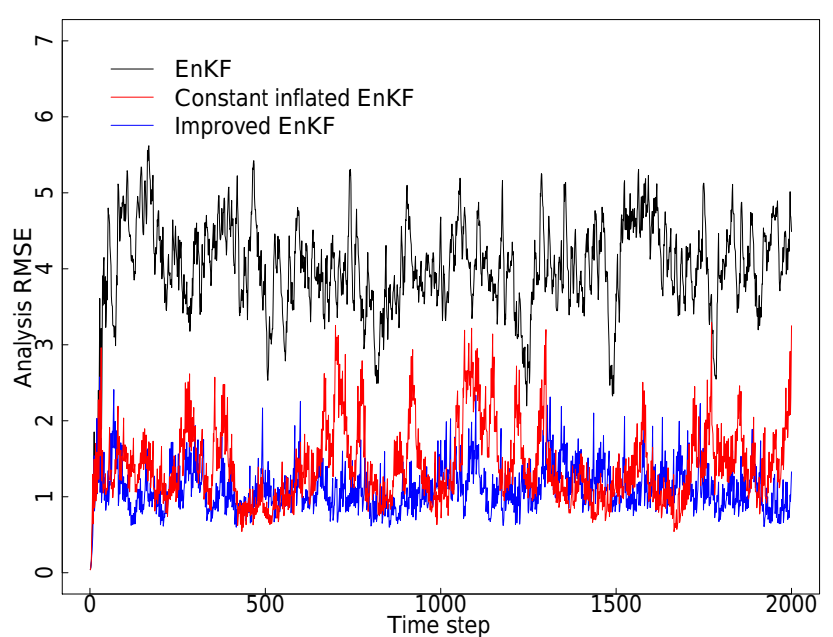

Figure 5. Analysis RMSE of the conventional EnKF (black line), the constant inflated EnKF (red line) and the improved EnKF (blue line) for the Lorenz-96 experiment with 40-observation and 30ensemble member. The constant multiplicative inflation factor is set as 1.88 .

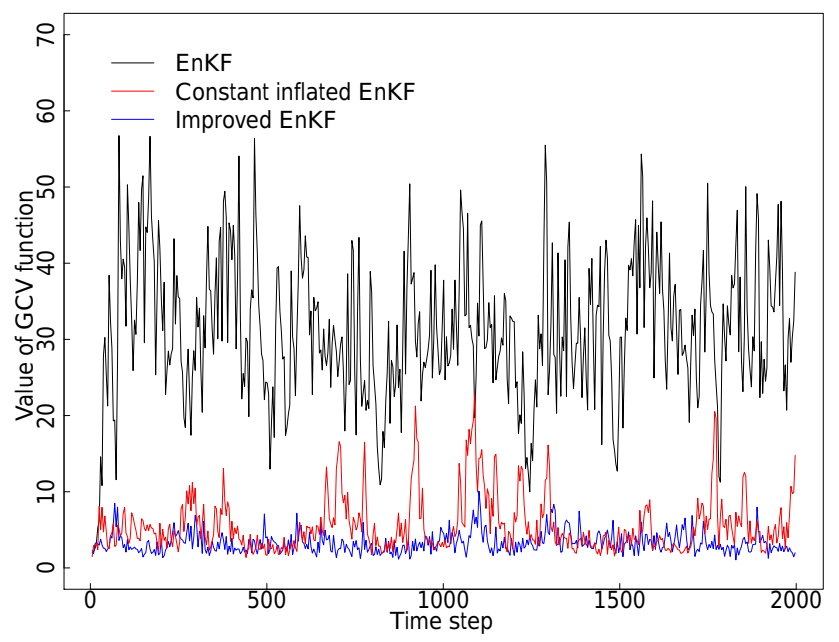

Figure 6. GCV function values of the conventional EnKF (black line), the constant inflated EnKF (red line) and the improved EnKF (blue line) for the Lorenz-96 experiment with 40-observation and 30 -ensemble member. The constant multiplicative inflation factor is set as 1.88 .

which can indeed increase the analysis sensitivity to the observations and reduce the analysis RMSE.

\subsection{Influence of ensemble size and observation number}

Intuitively, for any ensemble-based assimilation scheme, a large ensemble size will lead to small analysis errors; however, the computational costs are high for practical problems. The ensemble size in the practical land surface assimilation problem is usually several tens of members (Kirchgessner et al., 2014). The preferences of the proposed inflation method
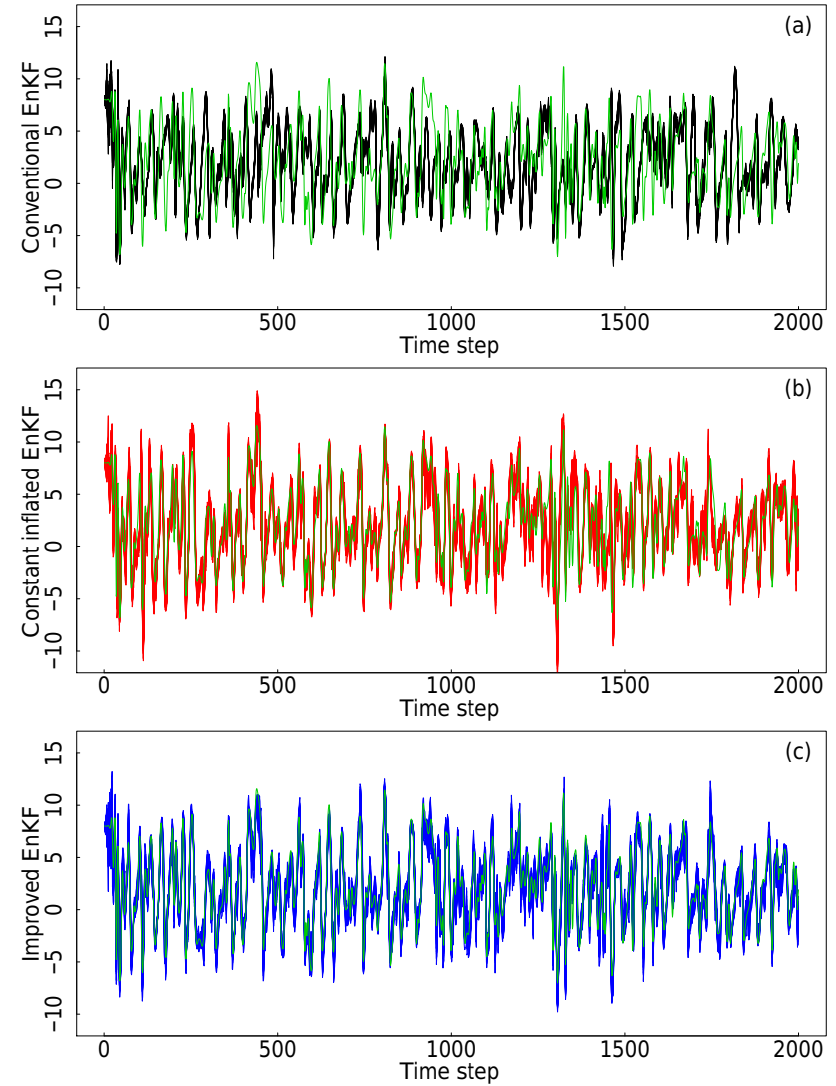

Figure 7. Ensemble analysis state members of the conventional EnKF (black line), the constant inflated EnKF (red line) and the improved EnKF (blue line) for the Lorenz-96 experiment with 40observation and 30-ensemble member. The constant multiplicative inflation factor is set as 1.88. The green line refers to the true trajectory obtained by the numerical solution.

and the constant inflation method with respect to different ensemble sizes (10, 30 and 50) were evaluated, and the results are listed in Table 1. It shows that for each scheme, using a 10-member ensemble produced a 3-fold increase in the analysis RMSE, while using a 50-member ensemble reduced the analysis RMSE by $20 \%$ relative to the analysis RMSE obtained using a 30-member ensemble. The forecast ensemble spread increased slightly from a 10-member ensemble to a 50-member ensemble. The GAI and GCV function values changed sharply from a 10 -member ensemble to a $30-$ member ensemble, and they became relatively stable from a 30-member ensemble to a 50-member ensemble. Ensembles less than 10 were unstable, and no significant changes occurred for ensembles greater than 50. Considering the computational costs for practical problems, a 30-member ensemble may be necessary for Lorenz-96 model to estimate statistically robust results. In the realistic problem, a system in which the errors grow in multiple directions will need more ensembles to produce statistically robust results. 
Table 1. Time-mean values of the forecast ensemble spread, GAI statistics, GCV functions and analysis RMSE over 2000 time steps, as well as the running times (second) for different assimilation schemes. The observation number is 40 and the ensemble size is selected as 10,30 and 50 , respectively.

\begin{tabular}{lrrrrrr}
\hline Scheme & $\begin{array}{r}\text { Ensemble } \\
\text { size }\end{array}$ & Spread & GAI & GCV & RMSE & $\begin{array}{r}\text { Running } \\
\text { time }\end{array}$ \\
\hline Conventional & 10 & 0.23 & $4.56 \%$ & 36.38 & 4.50 & 70.73 \\
EnKF & 30 & 0.36 & $10.78 \%$ & 31.14 & 4.01 & 215.92 \\
& 50 & 0.41 & $13.58 \%$ & 25.21 & 3.52 & 346.69 \\
\hline Constant & 10 & 3.15 & $4.78 \%$ & 35.91 & 4.38 & 77.41 \\
inflated & 30 & 3.25 & $27.48 \%$ & 5.56 & 1.41 & 238.25 \\
EnKF & 50 & 3.27 & $19.67 \%$ & 5.03 & 1.14 & 384.63 \\
\hline Improved & 10 & 3.26 & $5.24 \%$ & 35.56 & 3.74 & 81.31 \\
EnKF & 30 & 3.32 & $29.21 \%$ & 3.29 & 1.10 & 251.06 \\
& 50 & 3.45 & $35.63 \%$ & 2.30 & 0.88 & 405.68 \\
\hline
\end{tabular}

To evaluate the preferences of the inflation method with respect to different numbers of observations, synthetic observations were generated at every other grid point and for every 4 time steps. Hence, a total of 20 observations were performed at each observation step in this case. The assimilation results with ensemble sizes of 10,30 and 50 are listed in Table 2, which shows that the GAI values were larger than those with 40-observations in all assimilation schemes. This finding may be related to the relatively small denominator of the GAI statistic (Eq. 16) in the 20-observation experiments. The forecast ensemble spread does not change much but the GCV function and the RMSE values increase greatly in the 20-observation experiments with respect to those in the 40-observation experiments, which illustrates that more observations will lead to less analysis error.

\section{Discussions}

\subsection{Performance of the GCV inflation}

Accurate estimates of the forecast error covariance matrix are crucial to the success of any data assimilation scheme. In the conventional EnKF assimilation scheme, the forecast error covariance matrix is estimated as the sampling covariance matrix of the ensemble forecast states. However, limited ensemble size and large model errors often cause the matrix to be underestimated, which produces an analysis state that over relies on the forecast and excludes observations. This can eventually cause the filter to diverge. Therefore, the forecast error inflation with proper inflation factors is increasingly important.

The use of multiplicative covariance inflation techniques can mitigate this problem to some extent. Several methods have been proposed in the literature, and each has different assumptions. For instance, the moment approach can be easily conducted based on the moment estimation of the innovation statistic. The maximum likelihood approach can obtain a more accurate inflation factor than the moment approach, but requires computing high-dimensional matrix determinants. The Bayesian approach assumes a prior distribution for the inflation factor but is limited to spatially independent observational errors. In this study, the inflation factor was estimated based on cross-validation and the analysis sensitivity was detected. The estimated inflation factor by minimizing the GCV function is not affected by the observation unit and can optimize the analysis sensitivity to the observation.

In fact, the GCV method can evaluate and compare learning algorithms and represents a widely used statistical method. It can be applied in inverse problems in such fields as meteorological data assimilation (Wahba et al., 1995). Specifically, GCV provides a well-characterized method, which can select a regularization parameter by minimizing the predictive data errors with rotation-invariant in a leastsquares solution (MacCarthy et al., 2011). In data assimilation research fields, observation data such as in situ observation and remote sensing data are usually from different sources. GCV is particularly useful for choosing relative parameters that reflect not only measurement accuracies from different sources but also model capability (Krakauer et al., 2004). Apparently, GCV method requires calculating the trace of a large matrix, which may be commonly computationally prohibitive for large inverse problems (MacCarthy et al., 2011).

In this study, the GCV concept was adopted for the inflation factor estimation in the improved EnKF assimilation scheme and was validated with the Lorenz-96 model. The assimilation results showed that inflating the conventional EnKF using the factor estimated by minimizing the GCV function can indeed reduce the analysis RMSE. Therefore, the GCV function can accurately quantify the goodness of fit of the error covariance matrix. The values of the GCV function obviously decreased in the proposed approach compared the conventional EnKF and constant inflated EnKF schemes. The analysis RMSE of the proposed approach was also much 
Table 2. Same as in Table 1 but for 20 observations.

\begin{tabular}{lrrrrrr}
\hline Scheme & $\begin{array}{r}\text { Ensemble } \\
\text { size }\end{array}$ & Spread & GAI & GCV & RMSE & $\begin{array}{r}\text { Running } \\
\text { time }\end{array}$ \\
\hline Conventional & 10 & 0.41 & $10.77 \%$ & 33.64 & 4.85 & 67.75 \\
EnKF & 30 & 0.59 & $20.92 \%$ & 22.89 & 4.10 & 181.27 \\
& 50 & 0.68 & $26.41 \%$ & 14.97 & 3.29 & 295.92 \\
\hline Constant & 10 & 3.03 & $11.73 \%$ & 33.39 & 4.64 & 71.22 \\
inflated & 30 & 3.18 & $30.07 \%$ & 17.12 & 3.92 & 203.64 \\
EnKF & 50 & 3.27 & $39.51 \%$ & 12.74 & 3.37 & 322.29 \\
\hline Improved & 10 & 3.33 & $13.25 \%$ & 32.17 & 4.39 & 74.84 \\
EnKF & 30 & 3.36 & $35.09 \%$ & 14.99 & 3.46 & 213.81 \\
& 50 & 3.48 & $41.28 \%$ & 5.19 & 2.86 & 339.41 \\
\hline
\end{tabular}

smaller than those of the conventional EnKF and constant inflated EnKF schemes, which suggests that the GCV criterion works well for estimating the inflation factor.

The analysis sensitivities in the proposed approach and in the conventional EnKF scheme were also investigated in this study. The time-averaged GAI statistic increases from about $10 \%$ in the conventional EnKF scheme to about $30 \%$ using the proposed inflation method. This illustrates that the inflation mitigates the problem of the analysis depending excessively on the forecast and excluding the observations. The relationship of the analysis state to the forecast state and the observations are more reasonable.

\subsection{Computational cost}

The highest computational cost when minimizing the GCV function is related to calculating the influence matrix $\mathbf{A}_{i}(\lambda)$. Since the matrix multiplication is commutative for the trace, the GCV function can be easily re-expressed as follows:

$\operatorname{GCV}_{i}(\lambda)=\frac{p_{i} \boldsymbol{d}_{i}^{\mathrm{T}}\left(\mathbf{H}_{i} \lambda \mathbf{P}_{i} \mathbf{H}_{i}^{\mathrm{T}}+\mathbf{R}_{i}\right)^{-1} \mathbf{R}_{i}\left(\mathbf{H}_{i} \lambda \mathbf{P}_{i} \mathbf{H}_{i}^{\mathrm{T}}+\mathbf{R}_{i}\right)^{-1} \boldsymbol{d}_{i}}{\left[\operatorname{Tr}\left(\left(\mathbf{H}_{i} \lambda \mathbf{P}_{i} \mathbf{H}_{i}^{\mathrm{T}}+\mathbf{R}_{i}\right)^{-1} \mathbf{R}_{i}\right)\right]^{2}}$.

Because both the numerator and denominator of the GCV function are scalars, the inverse matrix is needed only in $\left(\mathbf{H}_{i} \lambda \mathbf{P}_{i} \mathbf{H}_{i}^{\mathrm{T}}+\mathbf{R}_{i}\right)^{-1}$, which can be effectively calculated using the Sherman-Morrison-Woodbury formula. Furthermore, the inverse matrix calculation and the multiplication process are also indispensable for the conventional EnKF (Eq. 6). Essentially, no additional computational burden is associated with the improved EnKF for the inverse matrix. Therefore, the total computational costs of the improved EnKF are feasible.

For the Lorenz-96 experiments in this study, the conventional EnKF, constant inflated EnKF and proposed improved EnKF assimilation schemes were conducted using $R$ language on a computer with Intel Core i5 CPU and 8 GB RAM. The running times with different observation numbers and ensemble sizes were listed in Tables 1 and 2. It shows that for each assimilation scheme, the computational cost increases as the ensemble size grows. For the fixed observation number and ensemble size, the conventional EnKF, which does not involve the forecast error inflation, has the least running time but at a cost of losing assimilation accuracy. The proposed EnKF scheme is about $15 \%$ smaller in analysis RMSE, but only about $5 \%$ longer in running time than the constant inflated EnKF scheme. For the operational meteorological/ocean models, the most computational cost is in the ensemble model integrations (Ravazzani et al., 2016). Therefore, the proposed EnKF scheme does not significantly increase computational cost.

\subsection{Notes}

It is worth noting that the inflation factor is assumed to be constant in space in this study, which may be not the case in realistic assimilation problems. Forcing all components of the state vector to use the same inflation factor could systematically overinflate the ensemble variances in sparsely observed areas, especially when the observations are unevenly distributed. In the presence of sparse observations, the state that is not observed can be improved only by the physical mechanism of the forecast model, although this improvement is limited. Therefore, a multiplicative inflation may not be sufficiently effective to enhance the assimilation accuracy. In this case, the additive inflation and the localization technique can be applied to further improve the assimilation quality in the presence of sparse observations (Miyoshi and Kunii, 2011; Yang et al., 2015).

\section{Conclusions}

In this study, the approach for using GCV as a metric to estimate the covariance inflation factor was proposed. In the case studies conducted in Sect. 3, the observations were relatively evenly distributed and the assimilation accuracy could indeed be improved by the forecast error inflation technique. These findings provide insights on the methodology and val- 
idation of the Lorenz-96 model and illustrate the feasibility Data availability. No data sets were used in this article. of our approach. In the near future, methods of modifying the adaptive procedure to suit the system with unevenly distributed observations and applying to more sophisticated dynamic and observation systems will be investigated. 


\section{Appendix A}

From Eq. (2), the normalized observation equation can be defined as follows:

$\widetilde{\boldsymbol{y}}_{i}^{\mathrm{o}}=\mathbf{R}_{i}^{-1 / 2} \mathbf{H}_{i} \boldsymbol{x}_{i}^{\mathrm{t}}+\widetilde{\boldsymbol{\varepsilon}}_{i}$,

where $\widetilde{\boldsymbol{y}}_{i}^{\mathrm{o}}=\mathbf{R}_{i}^{-1 / 2} \boldsymbol{y}_{i}^{\mathrm{o}}$ is the normalized observation vector and $\widetilde{\boldsymbol{\varepsilon}}_{i} \sim N(\mathbf{0}, \mathbf{I}) ; \mathbf{I}_{p_{i}}$ is the identity matrix with the dimensions $p_{i} \times p_{i}$. Similarly, the normalized analysis vector is $\widetilde{\boldsymbol{y}}_{i}^{\mathrm{a}}=\mathbf{R}_{i}^{-1 / 2} \mathbf{H}_{i} \boldsymbol{x}_{i}^{\mathrm{a}}$ and the influence matrix $\mathbf{A}_{i}$ relates the normalized observation vector to the normalized analysis vector, thereby ignoring the normalized forecast state in the observation space $(\mathrm{Gu}, 2002)$ :

$\tilde{\boldsymbol{y}}_{i}^{\mathrm{a}}-\mathbf{R}_{i}^{-1 / 2} \mathbf{H}_{i} \boldsymbol{x}_{i}^{\mathrm{f}}=\mathbf{A}_{i}\left(\widetilde{\boldsymbol{y}}_{i}^{\mathrm{o}}-\mathbf{R}_{i}^{-1 / 2} \mathbf{H}_{i} \boldsymbol{x}_{i}^{\mathrm{f}}\right)$.

Because the analysis state $\boldsymbol{x}_{i}^{\mathrm{a}}$ is given by Eq. (5), the influence matrix $\mathbf{A}_{i}$ can be verified as follows:

$\mathbf{A}_{i}=\mathbf{I}_{p_{i}}-\mathbf{R}_{i}^{1 / 2}\left(\mathbf{H}_{i} \mathbf{P}_{i} \mathbf{H}_{i}^{\mathrm{T}}+\mathbf{R}_{i}\right)^{-1} \mathbf{R}_{i}^{1 / 2}$.

If the initial forecast error covariance matrix is inflated as described in Sect. 2.2, then the influence matrix is treated as the following function of $\lambda$

$\mathbf{A}_{i}(\lambda)=\mathbf{I}_{p_{i}}-\mathbf{R}_{i}^{1 / 2}\left(\mathbf{H}_{i} \lambda \mathbf{P}_{i} \mathbf{H}_{i}^{\mathrm{T}}+\mathbf{R}_{i}\right)^{-1} \mathbf{R}_{i}^{1 / 2}$,

The principle of $\mathrm{CV}$ is to minimize the estimated error at the observation grid point. Lacking an independent validation data set, a common alternative strategy is to minimize the squared distance between the normalized observation value and the analysis value while not using the observation on the same grid point, which is the following objective function:

$V_{i}(\lambda)=\frac{1}{p_{i}} \sum_{k=1}^{p_{i}}\left(\tilde{\boldsymbol{y}}_{i, k}^{\mathrm{o}}-\left(\mathbf{R}_{i}^{-1 / 2} \mathbf{H}_{i} \boldsymbol{x}_{i}^{\mathrm{a}[k]}\right)_{k}\right)^{2}$,

where $x_{i}^{\mathrm{a}[k]}$ is the minima of the following "delete-one" objective function:

$$
\begin{aligned}
& \left(\boldsymbol{x}-\boldsymbol{x}_{i}^{\mathrm{f}}\right)^{\mathrm{T}}\left(\lambda \mathbf{P}_{i}\right)^{-1}\left(\boldsymbol{x}-\boldsymbol{x}_{i}^{\mathrm{f}}\right) \\
& \quad+\left(\boldsymbol{y}_{i}^{\mathrm{o}}-\mathbf{H}_{i} \boldsymbol{x}\right)_{-k}^{\mathrm{T}} \mathbf{R}_{i,-k}^{-1 / 2}\left(\boldsymbol{y}_{i}^{\mathrm{o}}-\mathbf{H}_{i} \boldsymbol{x}\right)_{-k} .
\end{aligned}
$$

The subscript $-k$ indicates a vector (matrix) with its $k$ th element ( $k$ th row and column) deleted. Instead of minimizing Eq. (A6) $p_{i}$ times, the objective function (Eq. A5) has another more simple expression $(\mathrm{Gu}, 2002)$ :

$V_{i}(\lambda)=\frac{1}{p_{i}} \sum_{k=1}^{p_{i}} \frac{\left(\widetilde{\boldsymbol{y}}_{i, k}^{\mathrm{o}}-\left(\mathbf{R}_{i}^{-1 / 2} \mathbf{H}_{i} \boldsymbol{x}_{i}^{\mathrm{a}}\right)_{k}\right)^{2}}{\left(1-a_{k, k}\right)^{2}}$,

where $a_{k, k}$ is the element at the site pair $(k, k)$ of the influence matrix $\mathbf{A}_{i}(\lambda)$. Then, $a_{k, k}$ is substituted with the average $\frac{1}{p_{i}} \sum_{k=1}^{p_{i}} a_{k, k}=\frac{1}{p_{i}} \operatorname{Tr}\left(\mathbf{A}_{i}(\lambda)\right)$ and the constant is ignored to obtain the following GCV statistic ( $\mathrm{Gu}, 2002)$ :

$\operatorname{GCV}_{i}(\lambda)=\frac{\frac{1}{p_{i}} \boldsymbol{d}_{i}^{\mathrm{T}} \mathbf{R}_{i}^{-1 / 2}\left(\mathbf{I}_{p_{i}}-\mathbf{A}_{i}(\lambda)\right)^{2} \mathbf{R}_{i}^{-1 / 2} \boldsymbol{d}_{i}}{\left[\frac{1}{p_{i}} \operatorname{Tr}\left(\mathbf{I}_{p_{i}}-\mathbf{A}_{i}(\lambda)\right)\right]^{2}}$.

\section{Appendix B}

The sensitivities of the analysis to the observation are defined as follows:

$\mathbf{S}_{i}^{\mathrm{o}}=\frac{\partial \widetilde{\boldsymbol{y}}_{i}^{\mathrm{a}}}{\partial \widetilde{\boldsymbol{y}}_{i}^{\mathrm{o}}}=\mathbf{R}_{i}^{1 / 2} \mathbf{K}_{i}^{\mathrm{T}} \mathbf{H}_{i}^{\mathrm{T}} \mathbf{R}_{i}^{-1 / 2}$,

Substitute the Kalman gain matrix $\mathbf{K}_{i}=$ $\mathbf{P}_{i} \mathbf{H}_{i}^{\mathrm{T}}\left(\mathbf{H}_{i} \mathbf{P}_{i} \mathbf{H}_{i}^{\mathrm{T}}+\mathbf{R}_{i}\right)^{-1}$ into $\mathbf{S}_{i}^{\mathrm{o}}$, then:

$$
\begin{aligned}
\mathbf{S}_{i}^{\mathrm{o}}= & \mathbf{R}_{i}^{1 / 2} \mathbf{K}_{i}^{\mathrm{T}} \mathbf{H}_{i}^{\mathrm{T}} \mathbf{R}_{i}^{-1 / 2} \\
= & \mathbf{R}_{i}^{1 / 2}\left(\mathbf{H}_{i} \mathbf{P}_{i} \mathbf{H}_{i}^{\mathrm{T}}+\mathbf{R}_{i}\right)^{-1} \mathbf{H}_{i} \mathbf{P}_{i} \mathbf{H}_{i}^{\mathrm{T}} \mathbf{R}_{i}^{-1 / 2} \\
= & \mathbf{R}_{i}^{1 / 2}\left(\mathbf{H}_{i} \mathbf{P}_{i} \mathbf{H}_{i}^{\mathrm{T}}+\mathbf{R}_{i}\right)^{-1}\left(\mathbf{H}_{i} \mathbf{P}_{i} \mathbf{H}_{i}^{\mathrm{T}}+\mathbf{R}_{i}-\mathbf{R}_{i}\right) \mathbf{R}_{i}^{-1 / 2} \\
= & \mathbf{R}_{i}^{1 / 2}\left(\mathbf{H}_{i} \mathbf{P}_{i} \mathbf{H}_{i}^{\mathrm{T}}+\mathbf{R}_{i}\right)^{-1}\left(\mathbf{H}_{i} \mathbf{P}_{i} \mathbf{H}_{i}^{\mathrm{T}}+\mathbf{R}_{i}\right) \mathbf{R}_{i}^{-1 / 2} \\
& -\mathbf{R}_{i}^{1 / 2}\left(\mathbf{H}_{i} \mathbf{P}_{i} \mathbf{H}_{i}^{\mathrm{T}}+\mathbf{R}_{i}\right)^{-1} \mathbf{R}_{i} \mathbf{R}_{i}^{-1 / 2} \\
= & \mathbf{I}_{p_{i}}-\mathbf{R}_{i}^{1 / 2}\left(\mathbf{H}_{i} \lambda \mathbf{P}_{i} \mathbf{H}_{i}^{\mathrm{T}}+\mathbf{R}_{i}\right)^{-1} \mathbf{R}_{i}^{1 / 2} \\
= & \mathbf{A}_{i} .
\end{aligned}
$$

Therefore, the sensitivity matrix $\mathbf{S}_{i}^{\mathrm{o}}$ is equal to the influence matrix $\mathbf{A}_{i}$. 
Competing interests. The authors declare that they have no conflict of interest.

Acknowledgements. This work is supported by the National Natural Science Foundation of China (grant no. 91647202), the National Basic Research Program of China (grant no. 2015CB953703), the National Natural Science Foundation of China (grant no. 41405098) and the Fundamental Research Funds for the Central Universities. The authors would like to gratefully acknowledge the two anonymous reviewers and the editor for their constructive comments, which helped significantly in improving the quality of this manuscript.

Edited by: Amit Apte

Reviewed by: two anonymous referees

\section{References}

Allen, D. M.: The relationship between variable selection and data augmentation and a method for prediction, Technometrics, 16, 125-127, 1974.

Anderson, J. L.: An adaptive covariance inflation error correction algorithm for ensemble filters, Tellus A, 59, 210-224, 2007.

Anderson, J. L.: Spatially and temporally varying adaptive covariance inflation for ensemble filters, Tellus A, 61, 72-83, 2009.

Anderson, J. L. and Anderson, S. L.: A Monte Carlo implementation of the nonlinear fltering problem to produce ensemble assimilations and forecasts, Mon. Weather Rev., 127, 2741-2758, 1999.

Burgers, G., Leeuwen, P. J., and Evensen, G.: Analysis scheme in the ensemble kalman filter, Mon. Weather Rev., 126, 1719-1724, 1998.

Butcher, J. C.: Numerical methods for ordinary differential equations, John Wiley \& Sons, Chichester, 425 pp., 2003.

Cardinali, C., Pezzulli, S., and Andersson, E.: Influence - matrix diagnostic of a data assimilation system, Q. J. Roy. Meteor. Soc., 130, 2767-2786, 2004.

Constantinescu, E. M., Sandu, A., Chai, T., and Carmichael, G. R.: Ensemble-based chemical data assimilation I: general approach, Q. J. Roy. Meteor. Soc., 133, 1229-1243, 2007.

Craven, P. and Wahba, G.: Smoothing noisy data with spline functions, Numer. Math., 31, 377-403, 1979.

Dee, D. P.: On-line estimation of error covariance parameters for atmospheric data assimilation, Mon. Weather Rev., 123, 1128$1145,1995$.

Dee, D. P. and Silva, A. M.: Maximum-likelihood estimation of forecast and observation error covariance parameters part I: methodology, Mon. Weather Rev., 127, 1822-1834, 1999.

Ellison, C. J., Mahoney, J. R., and Crutchfield, J. P.: Prediction, Retrodiction, and the Amount of Information Stored in the Present, J. Stat. Phys., 136, 1005-1034, 2009.

Eubank, R. L.: Nonparametric regression and spline smoothing, Marcel Dekker, Inc., New York, 338 pp., 1999.

Evensen, G.: Sequential data assimilation with a nonlinear quasigeostrophic model using Monte Carlo methods to forecast error statistics, J. Geophys. Res., 99, 10143-10162, 1994.
Gentle, J. E., Hardle, W., and Mori, Y.: Handbook of computational statistics: concepts and methods, Springer, Berlin, 1070 pp., 2004.

Golub, G. H. and Loan, C. F. V.: Matrix Computations, The Johns Hopkins University Press: Baltimore, 1996.

Green, P. J. and Silverman, B. W.: Nonparametric Regression and Generalized Linear Models: A roughness penalty approach, Vol. 182, Chapman and Hall, London, 1994.

Gu, C.: Smoothing Spline ANOVA Models, Springer-Verlag, New York, 289 pp., 2002.

Gu, C. and Wahba, G.: Minimizing GCV/GML scores with multiple smoothing parameters via the Newton method, SIAM Journal on Scientific and Statistical Computation, 12, 383-398, 1991.

Ide, K., Courtier, P., Ghil, M., and Lorenc, A. C.: Unified notation for data assimilation operational sequential and variational, J. Meteorol. Soc. Jpn., 75, 181-189, 1997.

Kirchgessner, P., Berger, L., and Gerstner, A. B.: On the choice of an optimal localization radius in ensemble Kalman filter methods, Mon. Weather Rev., 142, 2165-2175, 2014.

Krakauer, N. Y., Schneider, T., Randerson, J. T., and Olsen, S. C.: Using generalized cross-validation to select parameters in inversions for regional carbon fluxes, Geophys. Res. Lett., 31, L19108, https://doi.org/10.1029/2004GL020323, 2004.

Li, H., Kalnay, E., and Miyoshi, T.: Simultaneous estimation of covariance inflatioin and observation errors within an ensemble Kalman filter, Q. J. Roy. Meteor. Soc., 135, 523-533, 2009.

Liang, X., Zheng, X., Zhang, S., Wu, G., Dai, Y., and Li, Y.: Maximum Likelihood Estimation of Inflation Factors on Error Covariance Matrices for Ensemble Kalman Filter Assimilation, Q. J. Roy. Meteor. Soc., 138, 263-273, 2012.

Liu, J., Kalnay, E., Miyoshi, T., and Cardinali, C.: Analysis sensitivity calculation in an ensemble Kalman filter, Q. J. Roy. Meteor. Soc., 135, 1842-1851, 2009.

Lorenz, E. N.: Predictability - a problem partly solved, Seminar on Predictability, ECMWF: Reading, UK, 1996.

Lorenz, E. N. and Emanuel, K. A.: Optimal sites for supplementary weather observations simulation with a small model, J. Atmos. Sci., 55, 399-414, 1998.

MacCarthy, J. K., Borchers, B., and Aster, R. C.: Efficient stochastic estimation of the model resolution matrix diagonal and generalized cross-validation for large geophysical inverse problems, J. Geophys. Res., 116, B10304, https://doi.org/10.1029/2011JB008234, 2011.

Miller, R. N., Ghil, M., and Gauthiez, F.: Advanced data assimilation in strongly nonlinear dynamical systems, J. Atmos. Sci., 51, 1037-1056, 1994.

Miyoshi, T.: The Gaussian approach to adaptive covariance inflation and its implementation with the local ensemble transform Kalman filter, Mon. Weather Rev., 139, 1519-1534, 2011.

Miyoshi, T. and Kunii, M.: The Local Ensemble Transform Kalman Filter with the Weather Research and Forecasting Model: Experiments with Real Observations, Pure Appl. Geophys., 169, 321333, 2011.

Pena, D. and Yohai, V. J.: The detection of influential subsets in linear regression using an influence matrix, J. Roy. Stat. Soc., 57, 145-156, 1991.

Ravazzani, G., Amengual, A., Ceppi, A., Homar, V., Romero, R., Lombardi, G., and Mancini, M.: Potentialities of ensemble strate- 
gies for flood forecasting over the Milano urban area, J. Hydrol., 539, 237-253, 2016.

Reichle, R. H.: Data assimilation methods in the Earth sciences, Adv. Water Resour., 31, 1411-1418, 2008.

Saltelli, A., Tarantola, S., Campolongo, F., and Ratto, M.: Sensitivity Analysis in Practice: A Guide to Assessing Scientific Models, John Wiley \& Sons, Chichester, 219 pp., 2004.

Saltelli, A., Ratto, A. M., Anders, T., Campolongo, F., Cariboni, J., Gatelli, D., Saisana, M., and Tarantola, S.: Global Sensitivity Analysis: The Primer. John Wiley \& Sons, Ispra, 292 pp., 2008.

Talagrand, O.: Assimilation of Observations, an Introduction, J. Meteorol. Soc. Jpn., 75, 191-209, 1997.

Tippett, M. K., Anderson, J. L., Bishop, C. H., Hamill, T. M., and Whitaker, J. S.: Notes and correspondence ensemble square root filter, Mon. Weather Rev., 131, 1485-1490, 2003.

Wahba, G. and Wold, S.: A completely automatic french curve, Commun. Stat., 4, 1-17, 1975.

Wahba, G., Johnson, D. R., Gao, F., and Gong, J.: Adaptive tuning of numerical weather prediction models randomized GCV in three- and four-dimensional data assimilation, Mon. Weather Rev., 123, 3358-3369, 1995.

Wand, M. P. and Jones, M. C.: Kernel Smoothing, Chapman and Hall, Maryland, 212 pp., 1995.

Wang, X. and Bishop, C. H.: A comparison of breeding and ensemble transform kalman filter ensemble forecast schemes, J. Atmos. Sci., 60, 1140-1158, 2003.
Wu, G., Zheng, X., Wang, L., Zhang, S., Liang, X., and Li, Y.: A New Structure for Error Covariance Matrices and Their Adaptive Estimation in EnKF Assimilation, Q. J. Roy. Meteor. Soc., 139, 795-804, 2013.

Wu, G., Yi, X., Wang, L., Liang, X., Zhang, S., Zhang, X., and Zheng, X.: Improving the ensemble transform Kalman filter using a second-order Taylor approximation of the nonlinear observation operator, Nonlin. Processes Geophys., 21, 955-970, https://doi.org/10.5194/npg-21-955-2014, 2014.

Xu, T., Gómez-Hernández, J. J., Zhou, H., and Li, L.: The power of transient piezometric head data in inverse modeling: An application of the localized normal-score EnKF with covariance inflation in a heterogenous bimodal hydraulic conductivity field, Adv. Water Resour., 54, 100-118, 2013.

Yang, S.-C., Kalnay, E., and Enomoto, T.: Ensemble singular vectors and their use as additive inflation in EnKF, Tellus A, 67, 26536, https://doi.org/10.3402/tellusa.v67.26536, 2015.

Zheng, X.: An adaptive estimation of forecast error statistic for Kalman filtering data assimilation, Adv. Atmos. Sci., 26, 154 160, 2009.

Zheng, X. and Basher, R.: Thin-plate smoothing spline modeling of spatial climate data and its application to mapping south Pacific rainfall, Mon. Weather Rev., 123, 3086-3102, 1995. 